\title{
Acute Diastolic Dysfunction: A New Entity Recognized by Transesophageal Echocardiography in Thoraco-abdominal Aortic Aneurysm Surgery
}

Authors: Ashraf A. Fayad MBBCh, FACC; Homer Yang MD, FRCP; * Claudio S. Cina MD, FRCSC, MSc; Gregory L. Bryson, MD, FRCPC, M.Sc; Howard Nathan MD, FRCP; Ashraf A. Fayad MBBCh, FACC

Affiliations: Department of Anesthesiology, University of Ottawa

* Department of Biostatistics and Epidemiology, McMaster University

Background. Diastolic dysfunction as a chronic phenomenon has been examined in patients with hypertension, coronary artery atherosclerosis, and pericardial diseases. Development of intraoperative diastolic dysfunction due to aortic cross clamp application in vascular surgery is an acute phenomenon that has not been previously described. The purpose of this study is to report transesophageal echocardiography (TEE) findings consistent with intraoperative acute diastolic dysfunction (ADD) in a series of patients undergoing thoracoabdominal aortic aneurysm (TAAA) repair.

Methods. This is a prospective cohort study of patients undergoing TAAA repair with intraoperative TEE monitoring. Patient demographics and pre-operative investigations were recorded. Surgical repair was performed with the adjunct of a left atrio-femoral bypass (LAFB). Invasive arterial and venous pressure monitoring were used. TEE was utilised to assess the diastolic function before and during aortic cross clamping. Diastolic function was measured using mitral inflow pulsed wave Doppler (E: A ratio).

Results. Nine patients with mean age of $67 \pm 5$ years underwent TAAA repair. All patients demonstrated E:A ratio $>1$ before aortic cross clamping. During aortic cross clamp, 6 of 9 patients (66\%) developed reversal of the E: A ratio consistent with ADD. Patients with the diastolic dysfunction were treated with nitroglycerine infusions, which resulted in normalization of the E: A ratio. The 3 patients who did not develop any E: A ratio changes were on B-blockers preoperatively.

Conclusions. While chronic diastolic dysfunction is a well-known entity, this is the first report of acute and reversible intra-operatve reversal of the E:A ratio suggesting ADD. 